\title{
Predicting Success with Silicon-Hydrogel Contact Lenses in New Wearers
}

Nigel Best, ${ }^{1,2}$ Laura Drury, James S.Wolffsohn.

1 Specsavers, 41 High Row, Darlington, Co Durham, DL3 7QW

2 Aston University, Life and Health Science, Ophthalmic Research Group, Birmingham, UK

\section{Corresponding author:}

Prof James Wolffsohn, Life and Health Science, Aston University, Aston Triangle, Birmingham, B4 7ET, UK Tel: $\quad+44(0) 121$ 2044140E-mail:

j.s.w.wolffsohn@aston.ac.uk 


\section{Abstract}

Purpose: to evaluate changes in tear metrics and ocular signs induced by six months of silicone-hydrogel contact lens wear and the difference in baseline characteristics between those who successfully continued in contact lens wear compared to those that did not. Methods: Non-invasive Keratograph, Tearscope and fluorescein tear break-up times (TBUTs), tear meniscus height, bulbar and limbal hyperaemia, lid-parallel conjunctival folds (LIPCOF), phenol red thread, fluorescein and lissamine-green staining, and lid wiper epitheliopathy were measured on 60 new contact lens wearers fitted with monthly siliconehydrogels (average age 36+14years, 40 females). Symptoms were evaluated by the Ocular Surface Disease Index . After six months full time contact lens wear the above metrics were re-measured on those patients still in contact lens wear $(n=33)$. The initial measurements were also compared between the group still wearing lenses after six months and those who had ceased lens wear $(n=27)$.

Results: There were significant changes in tear meniscus height ( $p=0.031)$, bulbar hyperaemia $(p=0.011)$, fluorescein TBUT $(p=0.027)$, corneal $(p=0.007)$ and conjunctival $(p=0.009)$ staining, LIPCOF $(p=0.011)$ and lid wiper epitheliopathy $(p=0.002)$ after six months of silicone-hydrogel wear. Successful wearers had a higher non-invasive (17.0s \pm $8.2 s$ vs $12.0 s \pm 5.6 s ; p=0.001)$ and fluorescein $(10.7 s \pm 6.4 s$ vs $7.5 s \pm 4.7 s ; p=0.001)$ TBUT than drop-outs, although OSDI (cut-off 4.2) was also a strong predictor of success.

Conclusion: Silicone-hydrogel lenses induced significant changes in the tear film and ocular surface and lid margin staining. Wettability of the ocular surface is the main factor affecting contact lens drop-out.

Keywords: contact lens induced dry-eye; drop-out; comfort; neophytes; silicone-hydrogel contact lenses; tear-film 


\section{INTRODUCTION}

Research suggests approximately half of current contact lens wearers suffer from dryness and discomfort, particularly towards the end of the day.[1] The symptoms described by these individuals are very similar to dry eye sufferers, leading to this condition being termed contact lens induced dry eye (CLIDE).[2] This inevitably leads to dissatisfaction and is the greatest cause of discontinuation of lens wear. ${ }_{[3[3] 4]}$ Prior to fitting their patients with contact lenses there are a number of tests available to the practitioner to assess the quality and quantity of tears, to allow advice to be given on an individual's suitability for contact lenses and to recommend the most appropriate modality. Traditionally these tests have included non-invasive break-up time (NIBUT), invasive fluorescein tear break-up time (TBUT), corneal and conjunctival staining, tear prism height measurement, phenol red test and various symptomatology questionnaires. Bulbar and limbal hyperaemia can give an indication of ocular surface health and more recently the degree of both lid parallel conjunctival folding (LIPCOF) and lid wiper epitheliopathy (LWE) have been added to the list of potential indicators of dry eye.[5[[][]]] LIPCOF graded at least grade 2 is likely to be associated with dry eye symptoms [8]

Early silicone-hydrogel contact lenses caused small but statistically significant changes in ocular physiology and symptomatology in new contact lens wearers over 18 months wear, but these were clinically insignificant.[0] However, no studies have examined the effect of subsequent generations of silicone-hydrogel materials in contact lens neophytes. Pult and colleagues (2008) examined 61 experienced contact lens wearers and concluded that those with dryness symptoms exhibited significantly more LWE and LIPCOF. ${ }_{[2]}$ LIPCOF sum severity scores were the most predictive of symptoms. A further study by these researcher in 2011 concluded that NIBUT, tear meniscus height (TMH), phenol red thread test, LIPCOF, and LWE were significantly, but moderately, related to OSDI scores; the 
strongest relationship was achieved by combining NIBUT with nasal LIPCOF.[5] A number of studies have found a relationship between lid wiper epitheliopathy and CLIDE in patients wearing either hydrogel and silicone-hydrogel contact lenses ${ }_{[6,7,10,11]}$. However, it is still not clear which clinical measures predict those new patients that will drop-out of contact lens wear.

Therefore this study assessed the effect that six months of contact lens wear by unselected new lens wearers had on their tear metrics and ocular health. It also examined the baseline characteristics of those who successfully completed 6 months wear compared with those that did not. 


\section{METHODS}

\section{Subjects}

Sixty subjects (average age $36 \pm 14$ years, range 18-67; 40 females) were recruited from the patients of a community optometric practice in the North East of England. Consent was obtained after explanation of the study and possible consequences of taking part. The study was approved by the ethical committee of Aston University and conformed to the tenets of the Declaration of Helsinki. The subjects were excluded from the study if they had diabetes, Sjogrens Syndrome, recent ocular infection, allergy, any systemic or topical medications known to adversely affect the ocular surface or were pregnant. None of the subjects had ever worn contact lenses previously and all had requested to be fitted with contact lenses. They all expressed a desire to wear lenses full time and agreed to wear their lenses for a minimum of 6 hours per day for at least 6 days per week throughout the study.

\section{Contact Lens Fitting}

Prior to contact lens fitting, each of the subjects had a number of tear metrics recorded (right eye data only was used for statistical analysis) and were then fitted bilaterally with Lotrafilcon B (Alcon, Fort Worth, Texas, USA) silicone hydrogel contact lenses in either spherical or toric $(n=22)$ form (Table 1$)$. They were instructed how to insert and remove their lenses as well as being taught appropriate cleaning procedures with Sauflon (Synergi, Twickenham, London, UK) contact lens care solution. They were instructed to return for a 2 week check, a 1 month check and a six month check. On the six month check all tear metrics were re-measured. 
Table 1: $\quad$ Specifications and properties of contact lens material used in the study

\begin{tabular}{|l|c|c|}
\hline Property & Air Optix Aqua & Air Optix Astigmatism \\
\hline Brand name & \multicolumn{2}{|c|}{ Lotrafilcon A } \\
\hline Manufacturer & \multicolumn{2}{|c|}{ Alcon } \\
\hline Water content (\%) & \multicolumn{2}{|c|}{$33 \%$} \\
\hline Base curve/diameter (mm) & Bi-aspheric & Back surface toric \\
\hline Design & \multicolumn{2}{|c|}{110} \\
\hline $\begin{array}{l}\text { Oxygen permeability (Fatt } \\
\text { units) }\end{array}$ & 0.08 & 0.112 \\
\hline $\begin{array}{l}\text { Centre thickness (mm) }-3.00 \\
\text { DS }\end{array}$ & \multicolumn{2}{|c|}{1} \\
\hline FDA group & \multicolumn{2}{|c|}{ Plasma Treatment } \\
\hline Surface treatment & DMA, TRIS, siloxane macromer \\
\hline Principal monomers & \multicolumn{2}{|c|}{} \\
\hline
\end{tabular}

DMA (N,N-dimethylacrylamide) TRIS (trimethylsiloxy silane);

\section{Clinical evaluation}

The tear film metrics, evaluated in the following sequence due to the invasive nature of some tests, were:

$\underline{\text { Non-invasive keratograph break-up time (NIKBUT) }}$

NIKBUT was first determined using the tear analysis software (version 2.73r19) on the Keratograph (Oculus, Optikgerate, Germany). The ‘Tear Film Scan' software, permits an automated, examiner independent technique for measuring NIKBUT. An illuminated ring pattern is projected onto the cornea in the form of a Placido disk consisting of 22 rings. Once the patient is correctly aligned, the software prompts the practitioner to ask the patient to blink twice. The second blink triggers the video recording and measurement. The measurement finishes when one of two events occurs; either the subject blinks or significant distortion of the reflected image of the Placido rings occurs. Three consecutive readings were taken separated by at least 60 s and the median recorded.[12]

\section{$\underline{\text { Non-invasive break up time (NIBUT) }}$}

NIBUT was determined using a Tearscope Plus (Keeler Ltd, Windsor, UK) with a fine grid 
insert. [13] This grid was observed by a practitioner for any disruption which would indicate tear film break up with the NIBUT recorded as the time measured, in seconds, between a complete blink and the first observed break in the tear film or unavoidable blink. Three consecutive readings separated by at least 60 s were taken and the median recorded.

\section{$\underline{\text { Tear meniscus height }(\mathrm{TMH})}$}

Tear meniscus height was measured in millimetres using the tear analysis software on the Keratograph at the centre of the lower lid to a precision of $0.01 \mathrm{~mm}$. Three consecutive readings separated by at least 60 s were taken and the median recorded.

\section{Bulbar and limbal hyperaemia}

Bulbar and limbal hyperaemia were evaluated through a slit lamp microscope using 16x magnification and a diffuse white light.[14] They were graded using the Cornea and Contact Lens Research Unit (CCLRU) grading scale interpolated in 0.1 increments.[15]

\section{Lid parallel conjunctival folds}

LIPCOF are folds in the lower conjunctiva parallel to the lower lid margin which have been shown to be predictive of dry eye symptoms in contact lens wearers.[16] They were evaluated without the instillation of fluorescein using a 2-3 mm wide vertical slit located along the temporal limbus at an angle between the observation and illumination system of 20-30 degrees, viewed at $25 x$ magnification. LIPCOF was graded using a 4 point scale. $[16]$

\section{Osmolarity}

Tear osmolarity was measured using the Tearlab (Tearlab Ltd, San Diego, CA, USA) determined by measuring the impedance of an electric current passed through a very small sample of tears ( $<50$ nanolitres). ${ }_{[21]}$ The tears of patients with dry eyes generally 
have a higher osmolarity than normal patients, this hyperosmolarity being a primary cause of the inflammation seen in dry eye patients resulting in both ocular discomfort and surface

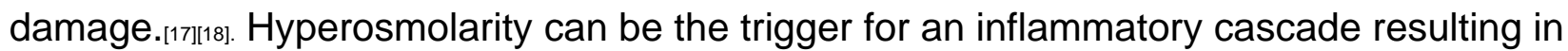
the production of inflammatory cytokines which can lead to increased apoptosis of corneal and conjunctival epithelial cells and conjunctival goblet cells.[19] A reduction in goblet cells will result in reduced mucin production ${ }_{[12]}$ and increased tear film instability (DEWS Report 2007).[22] A meta-analysis on published data for tear osmolarity showed a value of 316 mOsmol/L had a sensitivity of $59 \%$, specificity of $94 \%$ and a predictive accuracy of $89 \%$ for diagnosing dry eye disease. [23]

\section{Phenol red thread}

A cotton thread treated with the $\mathrm{pH}$ indicator phenol red (phenolsulfonphthalein) which is initially yellow in colour, but changes to light red on contact with tear fluid, was placed in the inferior temporal conjunctival sac and left in position for 15 seconds. The patient was instructed to look ahead and blink normally. The thread was then removed and the entire length of the red portion measured by a ruler to the nearest $0.5 \mathrm{~mm}$, including the folded section.[24]

\section{Fluorescein break up time (TBUT)}

Tear film TBUT was measured following the instillation of fluorescein into the temporal lower palpebral conjunctiva by a moist fluoret with excess saline shaken off. The cornea was then observed under blue light to excite the fluorescein molecules, through a yellow enhancement filter. ${ }_{[25]}$ The patient was instructed to blink and the time in seconds to the first observed tear film break-up or uncontrollable blink measured. Three consecutive readings separated by at least 60 s were taken and the median recorded. 


\section{Corneal staining}

Corneal staining was visualised under blue light to excite the fluorescein molecules, observed through a yellow enhancement filter to optimise visualisation following the instillation of fluorescein and classified using the CCLRU grading scale interpolated to 0.1 intervals. [15][25]

\section{Conjunctival staining}

Conjunctival staining was visualised through a Wratten 25 red filter following the instillation of lissamine green and classified using the CCLRU grading scale interpolated to 0.1 intervals. [15]

\section{Lid wiper epitheliopathy}

Following the instillation of the lissamine green the upper eyelid was everted and the length and sagittal width of any staining present was measured. The sagittal width of the lid wiper extends from just proximal to the line of Marx to the sub-tarsal fold. The staining was graded for length and width and the grades for these two characteristics were averaged for a final grade for LWE.[26] Care was taken to differentiate the staining associated with Marx's line from staining of the lid wiper.[9]

\section{Symptoms}

The OSDI questionnaire was used to measure patients' symptoms on their initial visit and again after 6 months of contact lens wear.[27] 


\section{Statistical Analyses}

Differences in tear metrics between the baseline and 6 month visits, and between those subjects who were still wearing contact lenses after 6 months and those who were not still wearing lenses after six months were analysed by performing either paired t-tests or the Wilcoxon t-test depending on whether the variables were normally distributed or not as assessed by the Kolmogorov-Smirnov test. The data was analysed using SPSS 18.0 software (IBM Corporation, New York, USA). A receiver operating curve of sensitivity and specificity for detecting those contact lens wearers dropping out from wear over the first 6 months was calculated. 


\section{Results}

\section{$\underline{\text { Relationship between tear metrics (baseline) }}$}

Measures of NIKBUT, NIBUT with the Tearscope and fluorescein break-up time tear stability tests were all related (Table 2). Tear quantity phenol red and TMH measures were also related. Limbal and bulbar hyperaemia were related to each other and interestingly to LIPCOF. LIPCOF was also related to fluorescein TBUT. Phenol red assessed tear volume was related to lissamine green conjunctival stain and LWE and conjunctival staining was the only metric related to subjective comfort as measured with the OSDI questionnaire (Table 2).

\section{Changes with 6 months lens wear}

Fluorescein TBUT, LIPCOF and TMH decreased over 6 months wear whereas bulbar hyperaemia, corneal and conjunctival staining and LWE increased (Table 3).

\section{Predictors of Drop out}

Twenty seven out of 60 neophyte patients had dropped out of contact lens wear by 6 months after fitting. Those who dropped out had a lower NIBUT and fluorescein TBUT at baseline than those who were still successfully wearing lenses (Table 3). 
Table 2: Correlation of tear film metrics at baseline $(n=60)$

\begin{tabular}{|c|c|c|c|c|c|c|c|c|c|c|c|c|c|}
\hline & NIKBUT & $\begin{array}{c}\text { Tearscope } \\
\text { NIBUT }\end{array}$ & $\begin{array}{c}\text { Fluores } \\
\text { cein }\end{array}$ & TMH & $\begin{array}{c}\text { Bulbar } \\
\text { hyperaemia }\end{array}$ & $\begin{array}{c}\text { Limbal } \\
\text { hyperaemia }\end{array}$ & LIPCOF & Osmolarity & $\begin{array}{c}\text { Phenol } \\
\text { Red }\end{array}$ & $\begin{array}{c}\text { Corneal } \\
\text { stain }\end{array}$ & $\begin{array}{l}\text { Conj } \\
\text { stain }\end{array}$ & LWE & OSDI \\
\hline NIKBUT & & $\begin{array}{l}r=0.427, \\
p=0.001\end{array}$ & $\begin{array}{l}r=0.256 \\
p=0.049\end{array}$ & $\begin{array}{l}r=- \\
0.109, \\
p=0.406\end{array}$ & $\begin{array}{l}r=-0.035, \\
p=0.694\end{array}$ & $\begin{array}{l}r=-0.052, \\
p=0.694\end{array}$ & $\begin{array}{l}r=0.069, \\
p=0.600\end{array}$ & $\begin{array}{l}r=-0.160 \\
p=0.221\end{array}$ & $\begin{array}{l}r=0.036, \\
p=0.0785\end{array}$ & $\begin{array}{l}r=- \\
0.036, \\
p=0.785\end{array}$ & $\begin{array}{l}r=- \\
0.075, \\
p=0.569\end{array}$ & $\begin{array}{l}r=- \\
0.095, \\
p=0.471\end{array}$ & $\begin{array}{l}r=0.078, \\
p=0.551\end{array}$ \\
\hline $\begin{array}{l}\text { Tearscope } \\
\text { NIBUT }\end{array}$ & & & $\begin{array}{l}r=0.550 \\
p<0.001\end{array}$ & $\begin{array}{l}r=- \\
0.100, \\
p=0.447\end{array}$ & $\begin{array}{l}r=-0.088, \\
p=0.503\end{array}$ & $\begin{array}{l}r=-0.102, \\
p=0.440\end{array}$ & $\begin{array}{l}r=- \\
0.0123 \\
p=0.348\end{array}$ & $\begin{array}{l}r=0.058, \\
p=0.661\end{array}$ & $\begin{array}{l}r=0.007, \\
p=0.955\end{array}$ & $\begin{array}{l}r=- \\
0.035, \\
p=0.789\end{array}$ & $\begin{array}{l}r=- \\
0.125, \\
p=0.341\end{array}$ & $\begin{array}{l}r=- \\
0.082, \\
p=0.534\end{array}$ & $\begin{array}{l}r=-0.125, \\
p=0.342\end{array}$ \\
\hline $\begin{array}{l}\text { Fluorescei } \\
\text { n TBUT }\end{array}$ & & & & $\begin{array}{l}r=0.153 \\
p=0.243\end{array}$ & $\begin{array}{l}r=-0.119, \\
p=0.366\end{array}$ & $\begin{array}{l}r=-0.086, \\
p=0.516\end{array}$ & $\begin{array}{l}r=- \\
0.257, \\
p=0.048\end{array}$ & $\begin{array}{l}r=0.061, \\
p=0.641\end{array}$ & $\begin{array}{l}r=0.011, \\
p=0.935\end{array}$ & $\begin{array}{l}r=- \\
0.187, \\
p=0.15\end{array}$ & $\begin{array}{l}r=0.018, \\
p=0.888\end{array}$ & $\begin{array}{l}r=- \\
0.201, \\
p=0.124\end{array}$ & $\begin{array}{l}r=-0.123, \\
p=0.348\end{array}$ \\
\hline TMH & & & & & $\begin{array}{l}r=0.198, \\
p=0.130\end{array}$ & $\begin{array}{l}r=0.200, \\
p=0.126\end{array}$ & $\begin{array}{l}\mathrm{r}=- \\
0.226, \\
p=0.083\end{array}$ & $\begin{array}{l}r=0.189, \\
p=0.147\end{array}$ & $\begin{array}{l}r=0.463, \\
p<0.001\end{array}$ & $\begin{array}{l}r=0.079, \\
p=0.546\end{array}$ & $\begin{array}{l}r=- \\
0.0100, \\
p=0.448\end{array}$ & $\begin{array}{l}r=- \\
0.208, \\
p=0.112\end{array}$ & $\begin{array}{l}r=0.002, \\
p=0.987\end{array}$ \\
\hline $\begin{array}{l}\text { Bulbar } \\
\text { hyperaemia }\end{array}$ & & & & & & $\begin{array}{l}r=0.715, \\
p<0.001\end{array}$ & $\begin{array}{l}r=0.466, \\
p<0.001\end{array}$ & $\begin{array}{l}r=-0.054, \\
p=0.682\end{array}$ & $\begin{array}{l}r=0.154, \\
p=0.241\end{array}$ & $\begin{array}{l}r=0.051, \\
p=0.696\end{array}$ & $\begin{array}{l}r=0.217, \\
p=0.095\end{array}$ & $\begin{array}{l}r=- \\
0.012, \\
p=0.929\end{array}$ & $\begin{array}{l}r=0.164, \\
p=0.210\end{array}$ \\
\hline $\begin{array}{l}\text { Limbal } \\
\text { hyperaemia }\end{array}$ & & & & & & & $\begin{array}{l}r=0.276, \\
p=0.033\end{array}$ & $\begin{array}{l}r=-0.163, \\
p=0.213\end{array}$ & $\begin{array}{l}r=0.340, \\
p=0.008\end{array}$ & $\begin{array}{l}r=0.107, \\
p=0.417\end{array}$ & $\begin{array}{l}r=0.184, \\
p=0.160\end{array}$ & $\begin{array}{l}r=0.048, \\
p=0.716\end{array}$ & $\begin{array}{l}r=-0.016, \\
p=0.903\end{array}$ \\
\hline LIPCOF & & & & & & & & $\begin{array}{l}r=-0.140, \\
p=0.286\end{array}$ & $\begin{array}{l}r=-0.084, \\
p=0.522\end{array}$ & $\begin{array}{l}r=- \\
0.054, \\
p=0.683\end{array}$ & $\begin{array}{l}r=0.249, \\
p=0.055\end{array}$ & $\begin{array}{l}r=0.211, \\
p-0.106\end{array}$ & $\begin{array}{l}r=0152, \\
p=0.248\end{array}$ \\
\hline Osmolarity & & & & & & & & & $\begin{array}{l}r=-0.233, \\
p=0.074\end{array}$ & $\begin{array}{l}r=0.164, \\
p=0.209\end{array}$ & $\begin{array}{l}r=0.220, \\
p=0.090\end{array}$ & $\begin{array}{l}r=0.152, \\
p=0.244\end{array}$ & $\begin{array}{l}r=0.036, \\
p=0.782\end{array}$ \\
\hline Phenol Red & & & & & & & & & & $\begin{array}{l}r=0.150, \\
p=0.254\end{array}$ & $\begin{array}{l}r=- \\
0.256, \\
p=0.048\end{array}$ & $\begin{array}{l}r=- \\
0.257, \\
p=0.048\end{array}$ & $\begin{array}{l}r=-0.055, \\
p=0.674\end{array}$ \\
\hline $\begin{array}{l}\text { Corneal } \\
\text { stain }\end{array}$ & & & & & & & & & & & $\begin{array}{l}r=0.038, \\
p=0.776\end{array}$ & $\begin{array}{l}r=- \\
0.083, \\
p=0.527\end{array}$ & $\begin{array}{l}r=0.112, \\
p=0.395\end{array}$ \\
\hline Conj stain & & & & & & & & & & & & $\begin{array}{l}r=0.032, \\
p=0.810\end{array}$ & $\begin{array}{l}r=0.273, \\
p=0.035\end{array}$ \\
\hline LWE & & & & & & & & & & & & & $\begin{array}{l}r=0.105, \\
p=0.426\end{array}$ \\
\hline
\end{tabular}


Table 3: Tear film metrics, how they change over 6 months wear of a silicone hydrogel in neophytes $(n=60)$ and the difference in baseline between those who are successful in lens wear $(n=33)$ and those that drop out $(n=27) . \pm=1$ S.D.

\begin{tabular}{|c|c|c|c|c|c|c|}
\hline Measure & $\begin{array}{l}\text { Normality (K- } \\
\text { S Z) }\end{array}$ & $\begin{array}{l}\text { Baseline of } \\
\text { Successful } \\
\text { Wearers }\end{array}$ & $\begin{array}{l}\text { After } 6 \text { months } \\
\text { Contact Lens } \\
\text { Wear }\end{array}$ & $\begin{array}{l}\text { Significanc } \\
\text { e with } \\
\text { Wear }\end{array}$ & $\begin{array}{l}\text { Baseline } \\
\text { of Drop- } \\
\text { outs }\end{array}$ & $\begin{array}{l}\text { Significance } \\
\text { with Success }\end{array}$ \\
\hline NIK-BUT (s) & 1.225, $p=0.099$ & $5.9 \pm 4.3$ & $6.2 \pm 3.5$ & 0.124 & $4.9 \pm 4.1$ & 0.920 \\
\hline NIBUT (s) & $1.334, p=0.057$ & $17.0 \pm 8.2$ & $16.9 \pm 7.8$ & 0.306 & $12.0 \pm 5.6$ & 0.001 \\
\hline $\begin{array}{l}\text { Fluorescein } \\
\text { TBUT (s) }\end{array}$ & $1.286, p=0.073$ & $10.7 \pm 6.4$ & $8.7 \pm 5.1$ & 0.027 & $7.5 \pm 4.7$ & 0.045 \\
\hline TMH (mm) & $0.867, p=0.440$ & $0.26 \pm 0.09$ & $0.24 \pm 0.07$ & 0.031 & $0.26 \pm 0.09$ & 0.689 \\
\hline $\begin{array}{l}\text { Bulbar } \\
\text { Hyperaemia }\end{array}$ & $0.882, p=0.419$ & $2.5 \pm 0.5$ & $2.7 \pm 0.3$ & 0.011 & $2.5 \pm 0.5$ & 0.093 \\
\hline $\begin{array}{l}\text { Limbal } \\
\text { Hyperaemia }\end{array}$ & $0.854, p=0.459$ & $2.3 \pm 0.5$ & $2.5 \pm 0.6$ & 0.184 & $2.3 \pm 0.7$ & 0.162 \\
\hline LIPCOF & $2.040, p<0.001$ & $1.2 \pm 1.1$ & $0.9 \pm 1.0$ & 0.011 & $1.5 \pm 1.3$ & 0.070 \\
\hline $\begin{array}{l}\text { Osmolarity } \\
\text { (mmol) }\end{array}$ & $0.764, p=0.603$ & $321 \pm 12$ & $323 \pm 16$ & 0.202 & $325 \pm 20$ & 0.514 \\
\hline $\begin{array}{l}\text { Phenol Red } \\
(\mathrm{mm})\end{array}$ & $0.609, p=0.852$ & $16.9 \pm 6.5$ & $19.8 \pm 9.5$ & 0.086 & $15.5 \pm 8.4$ & 0.778 \\
\hline $\begin{array}{l}\text { Corneal } \\
\text { Staining }\end{array}$ & $3.739, p<0.001$ & $0.21 \pm 0.51$ & $0.86 \pm 0.79$ & 0.007 & $0.24 \pm 0.58$ & 0.947 \\
\hline $\begin{array}{l}\text { Conjunctival } \\
\text { Staining }\end{array}$ & $3.424, p<0.001$ & $0.51 \pm 0.93$ & $1.69 \pm 1.22$ & 0.009 & $0.51 \pm 1.04$ & 0.954 \\
\hline LWE & $3.464, p<0.001$ & $0.3 \pm 0.7$ & $1.5 \pm 1.2$ & 0.002 & $0.7 \pm 2.0$ & 0.826 \\
\hline OSDI & $\begin{array}{l}1.362, p= \\
0.0502\end{array}$ & $7.6 \pm 10.2$ & $8.5 \pm 10.4$ & 0.349 & $12.2 \pm 9.2$ & 0.255 \\
\hline
\end{tabular}


Received Operating Curves (ROC) for each of the tear film metrics are plotted in figure 1.

Those metrics which differentiated successful wearers from drop-outs $(p<0.05)$ were NIBUT, fluorescein TBUT and subjective rating with the OSDI (Table 4). Using a NIBUT cut-off of $10.0 \mathrm{~s}$ (as identified from the ROC as giving the best balance between sensitivity (63\%) and specificity (76\%)), 7 out of the $24(29 \%)$ with a NIBUT less than this value successfully wore contact lenses beyond 6 months, whereas of the 28 that dropped-out, $17(61 \%)$ had a fluorescein TBUT less than 10.0 s. Fluorescein TBUT had a lower cut-off of $5.5 \mathrm{~s}$ (as identified from the ROC as giving the best balance between sensitivity (56\%) and specificity (82\%)), 6 out of the 21 (29\%) with a fluorescein TBUT less than this value successfully wore contact lenses beyond 6 months, whereas of the 28 that dropped-out, 15 (54\%) had a fluorescein TBUT less than 5.5 s. Finally, an OSDI score greater than 4.2 (as identified from the ROC as giving the best balance between sensitivity (78\%) and specificity (64\%)), 11 out of the 36 (31\%) with an OSDI greater than this value successfully wore contact lenses beyond 6 months, whereas of the 28 that dropped out, 25 (89\%) had an OSDI greater than 4.2 .

ROC for Drop-Out

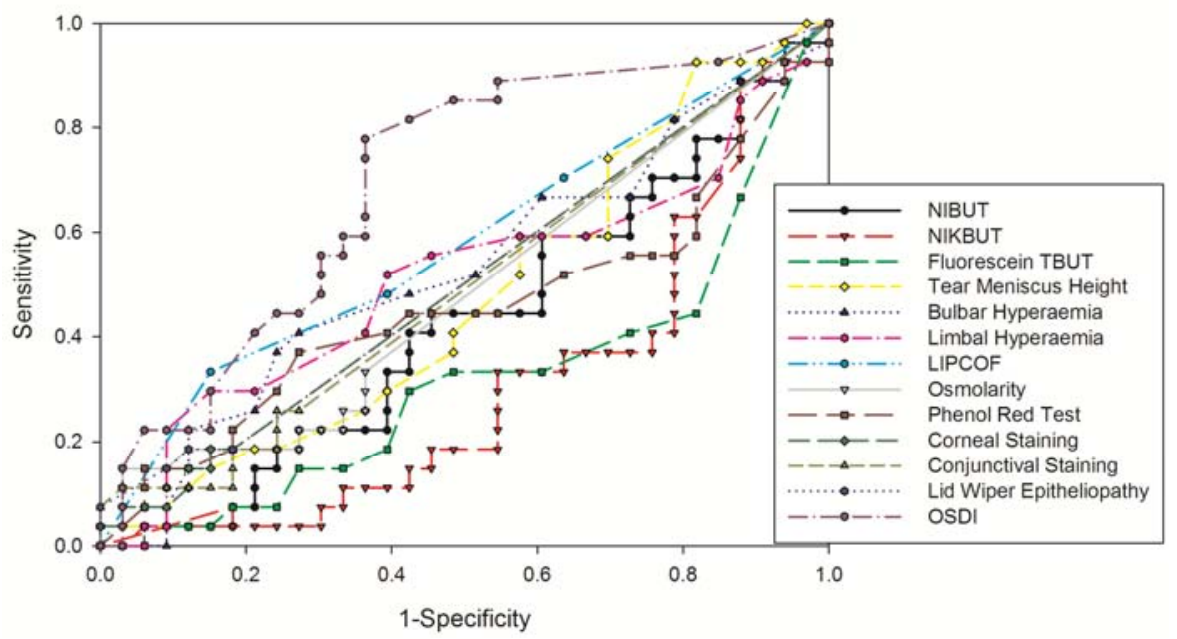

Figure 1: Receiver Operating Curves for each of the tear film metrics differentiating those that successfully wore contact lenses for 6 months $(N=33)$ compared to those that dropped out $(\mathrm{N}=27)$. 
Table 4: Tear film metrics and Receiver Operating Curve discrimination between those who are successful in lens wear $(n=33)$ and those that drop out $(n=27)$.

\begin{tabular}{|c|c|c|c|c|c|}
\hline \multirow[b]{2}{*}{ Tear Film Metrics } & \multirow[b]{2}{*}{ Area } & \multirow[b]{2}{*}{$\begin{array}{l}\text { Std. } \\
\text { Error }\end{array}$} & \multirow[b]{2}{*}{$\begin{array}{l}\text { Asymptotic } \\
\text { Sig. }\end{array}$} & \multicolumn{2}{|c|}{$\begin{array}{c}\text { Asymptotic 95\% Confidence } \\
\text { Interval }\end{array}$} \\
\hline & & & & Lower Bound & Upper Bound \\
\hline NIK-BUT (s) & .430 & .075 & .353 & .284 & .576 \\
\hline NIBUT (s) & .304 & .069 & .010 & .169 & .439 \\
\hline Fluorescein TBUT (s) & .320 & .071 & .017 & .181 & .458 \\
\hline TMH (mm) & .475 & .075 & .744 & .328 & .623 \\
\hline Bulbar Hyperaemia & .527 & .076 & .716 & .378 & .677 \\
\hline Limbal Hyperaemia & .509 & .078 & .905 & .356 & .662 \\
\hline LIPCOF & .577 & .075 & .305 & .430 & .725 \\
\hline Osmolarity (mmol) & .489 & .076 & .882 & .340 & .638 \\
\hline Phenol Red (mm) & .455 & .078 & .552 & .302 & .608 \\
\hline Corneal Staining & .503 & .076 & .964 & .355 & .652 \\
\hline Conjunctival Staining & .497 & .076 & .964 & .348 & .645 \\
\hline LWE & .511 & .076 & .882 & .362 & .660 \\
\hline OSDI & .694 & .069 & .010 & .558 & .829 \\
\hline
\end{tabular}




\section{Discussion}

The aim of this study was to assess the effect that six months of contact lens wear by unselected new lens wearers had on their tear metrics and ocular health and to examine the baseline characteristics of those who successfully completed 6 months wear compared with those that dropped out. The results of the study showed that NIKBUT, NIBUT with the Tearscope and fluorescein TBUT tear stability tests were all related (Table 2). This suggests that, rather than carrying out both an invasive and non-invasive measurement of tear film stability, one alone may suffice. Objective measures of NIBUT, such as the Keratograph, offer great potential to better understand localised drying of the ocular surface without subjectivity, but early software versions, such as used in this study, had limitations.[12] There was no significant difference in NIKBUT or NIBUT after 6 months of SiH contact lens wear; some previous studies with hydrogel lenses have shown similar results, [28-[29] while other studies have reported reduced NIBUT in hydrogel contact lens wearers.[30][31] There were clinically and statistically significant differences in both NIBUT (on average by $5.0 \mathrm{~s}$ ) and fluorescein TBUT (on average by $3.2 \mathrm{~s}$ ) between those subjects still wearing lenses after six months and those who had ceased lens wear. Receiver operating curves confirmed that this was a key metric to determine those neophyte patients likely to drop out of contact lens wear.

A tear meniscus height of less than $0.2 \mathrm{~mm}$ can indicate a reduced tear output and has been shown to correlate with contact lens intolerance. ${ }_{\text {[33] }}$ Therefore the correlation of $\mathrm{TMH}$ with phenol red test measured tear volume was expected, despite the lack of association found by Tomlinson and colleagues, although their study had fewer and slightly younger subjects. [34] Lid wiper epitheliopathy occurs when the cells along the upper lid margin are altered by the frictional forces which occur when the lid passes over the cornea or the front surface of a contact lens. ${ }_{[26]}$ According to Korb and colleagues ${ }_{[26]} 80 \%$ of symptomatic 
contact lens wearers will have staining of the lid wiper compared to only $13 \%$ of asymptomatic lens wearers. Lid wiper epitheliopathy was associated with tear volume, but not tear meniscus height. This might suggest that the tear volume remaining on the ocular surface is key to reducing the friction with the lid margin columnar cells, rather than the volume of the tear reservoir along the lower lid margin. However, lid wiper damage increased over 6 months of lens wear, whereas tear volume assessed by the phenol red test did not change, which does not support this explanation. Whilst there was a statistically significant decrease in $\mathrm{TMH}$ after six month of lens wear (on average by 0.02 $\mathrm{mm}$ ) this could be considered clinically insignificant. Tear volume as assessed by the phenol red test did not change over this time period, but lid wiper damage did significantly increase, tear volume as quantified by the tear meniscus height or phenol red test not aid the prediction of contact lens drop-out over 6 months wear, nor did the baseline presence of lid wiper damage.

Bulbar and limbal hyperaemia, along with LIPCOF, were found to be associated prior to lens fitting. An increase in bulbar hyperaemia was found over 6 months wear, but conversely a decrease in LIPCOF occurred. Whilst statistically significant the changes of on average 0.2 to 0.3 grading scale units were not felt to be clinically significant. Possible causes could include mechanical irritation from the lens ${ }_{[32]}$ or solution toxicity.[34] No significant difference in limbal hyperaemia was found despite being correlated to bulbar hyperaemia. Limbal hyperaemia can indicate corneal hypoxia and it has been shown before that eyes wearing silicone hydrogel lenses are less likely to show an increase in limbal redness.[36][37] None of these measures prior to lens wear predicted subsequent contact lens drop out.

A statistically significant increase in both corneal and conjunctival staining were found over 
6 months wear, which could be attributable to a number of factors including the mechanical effects of silicone hydrogel lenses ${ }_{[38]}$ and lens deposition. ${ }_{[39]}$ Prior studies have not shown a link between dry eye symptoms and corneal staining ${ }_{[40][41]}$ and patients in this study were found to have no drop in comfort over this period. Conjunctival staining was associated with the level of symptoms as has previously been shown.[42][43] Despite the lack of a significant difference between successful patients and contact lens drop outs based on the average comfort score (presumably due to the large variance between individuals in this subjective rating), the baseline OSDI was one of the best differentiators of patients likely to drop out. Interestingly, osmolarity was not found to be related to any of the other tear film metric quantified during this study, it did not change with lens wear and did not predict contact lens drop out, despite its inclusion in the definition of dry eyes.[22]

It is not surprising that the regular presence of a contact lens can cause changes in both tear metrics and ocular signs. These clinically significant changes in corneal and conjunctival staining, fluorescein TBUT and LWE were greater than might be expected from a previous study investigating the fitting of neophytes with early silicone hydrogel contact lenses, $[9]$ but indicate that contact lenses still need to be developed to achieve full biocompatibility. Care should be taken in fitting patients new to contact lenses if they have a NIBUT less than 10 s or an OSDI comfort rating greater than 4.2 as they are more likely to drop-out within the first 6 months. None of the other tear film metrics assessed were found to predict soft contact lens drop out. Fluorescein TBUT is strongly associated with NIBUT so its predictive abilities are largely redundant. The NIBUT and OSDI metrics are quick to obtain and can aid communication with the patient to examine other aspects related to contact lens wear success such as motivation ${ }_{[44-45]}$ and lens material properties [46-49] with an aim to reduce contact lens drop-out. [3][4] 


\section{References}

[1] Morgan PB, Efron N. Demographics of UK contact lens prescribing. Contact Lens Ant Eye 2008;31:50-1.

[2] Pult H, Purslow C, Murphy PJ. Clinical tests for successful contact lens wear: relationship and predictive potential. Optom Vis Sci 2008;85:924-9.

[3] Pritchard N, Fonn D, Brazeau D. Discontinuation of contact lens wear: a survey. Int Contact Lens Clin 1999;26:157-62.

[4] Richdale K, Sinnott LT, Skadahl E, Nichols JJ. Frequency of and factors associated with contact lens dissatisfaction and discontinuation. Cornea 2007;26:168-74.

[5] Pult H, Purslow C, Murphy PJ. The relationship between clinical signs and dry eye symptoms. Eye 2011;25):502-10.

[6] Yeniad B, Beginoglu M, Bilgin LK. Lid-wiper epitheliopathy in contact lens users and patients with dry eye. Eye Contact Lens 2010;36:140-3.

[7] Korb DR, Greiner JV, Herman JP, Hebert E, Finnemore VM, Exford JM, Glonek T, Olson MC. Lid-wiper epitheliopathy and dry-eye symptoms in contact lens wearers. CLAO J 2002;28:211-6.

[8] Nemeth J, Fodor E, Lang Z, Kosina-Hagyo K, Berta A, Komar T, Petricek I, Higazy M, Prost M, Grupcheva C, Kemer OE, Schollmayer P, Samaha A, Hlavackova K. Lidparallel conjunctival folds (LIPCOF) and dry eye: a multicentre study. $\mathrm{Br} \mathrm{J}$ Ophthalmol 2012;96:1380-5.

[9] Santodomingo-Rubido J, Wolffsohn J, Gilmartin B. Changes in ocular physiology, tear film characteristics, and symptomatology with 18 months silicone hydrogel contact lens wear. Optom Vis Sci 2006;83:73-81.

[10] Korb DR, Herman JP, Greiner JV, Scaffidi RC, Finnemore VM, Exford JM, Blackie CA, Douglass T. Lid wiper epitheliopathy and dry eye symptoms. Eye Contact Lens 2005;31:28.

[11] Pult H, Murphy PJ, Purslow C. A novel method to predict the dry eye symptoms in new contact lens wearers. Optom Vis Sci 2009;86:E1042-50.

[12] Best N, Drury L, Wolffsohn JS. Clinical evaluation of the Oculus Keratograph. Contact Lens Ant Eye 2012;35:171-4.

[13] Guillon JP. Use of the Tearscope Plus and attachments in the routine examination of the marginal dry eye contact lens patient. Adv Exp Med Biol 1998;438:859-67.

[14] Dundas M, walker A, Woods RL. Clinical grading of corneal staining of non-contact lens wearers. Ophthal Physiol Opt 2001;21:30-5. 
[15] Bailey IL, Bullimore MA, Raasch TW, Taylor HR. Clinical grading and the effects of scaling. Invest Ophthalmol Vis Sci 1991;32:422-32.

[16] Pult H, Purslow C, Berry C. The predictive ability of clinical tests for dry eye in contact lens wear Optom Vis Sci 2008;85:E924-9.

[17] Gilbard JP, Rossi SR, Santa Maria J. Osmolarity of tear microvolumes in keratoconjunctivitis sicca. Invest Ophthalmol Vis Sci 1978;28:225-8.

[18] Farris RL, Gilbard JP, Stuchell N, Mandell UD. Diagnostic tests in keratoconjunctivitis sicca. CLAO J 1983;9:23-8.

[19] Li DQ, Chen Z, Song XJ, Luo L, Pflugfelder SC. Stimulation of matrix metalloproteinases by hyperosmolarity via a JNK pathway in human corneal epithelial cells. Invest Ophthalmol Vis Sci 2004;45:4302-11

[20] Argueso P, Gipson IK. Epithelial mucins of the ocular surface:structure, biosynthesis and function. Exp Eye Res 2001;71:281-9.

[21] Sullivan B. Clinical reports of a first generation lab on chip nanolitre tear film osmometer. Ocul Surf 2005;3:s31

[22] DEWS report - international dry eye workshop (DEWS). Ocular Surface 2007;5:69193.

[23] Tomlinson A, Khanal S, Ramaesh K, Diaper C, McFadyen A. Tear Film Osmolarity: Determination of a Referent for Dry Eye Diagnosis. Invest Ophthalmol Vis Sci 2006;47:4309-15.

[24] Little SA, Bruce AS. Repeatability of the phenol-red thread and tear thinning time tests for tear film function. Clin Exp Optom 1994;77:64-8.

[25] Peterson,RC Wolffsohn,JS Fowler,CW. Optimization of Anterior Eye Fluorescein Viewing. Am J Ophthalmol 2006;142:572-5.

[26] Korb DR, Herman JP, Greiner JV, Scaffidi RC, Finnemore VM, Exford JM, Blackie CA, Douglass T. Lid Wiper Epitheliopathy and Dry Eye Symptoms. Eye Contact Lens 2005;31:2-8.

[27] Schiffman RM, Christianson MD, Jacobsen G, Hirsch JD, Reis BL. Reliability and validity of the Ocular Surface Disease Index. Arch Ophthalmol 2000;118:615-21.

[28] Cho P, Yap M. The effects of contact lens wear on the precorneal tear film of Chinese eyes. J BCLA 1995;18:87-94.

[29] Chui WS, Cho P, Brown B. Soft contact lens wear in Hong Kong-Chinese: predicting success . Ophthalmic Physiol Opt 2000;20:480-6.

[30] Du Toit R, Situ P, Simpson T, Fonn D. The effects of six months of contact lens wear on the tear film, ocular surfaces, and symptoms of presbyopes. Optom Vis Sci 
2001;78:455-62.

[31] Faber E, Golding TR, Lowe R, Brennan NA. Effect of hydrogel lens wear on tear film stability. Optom Vis Sci 1991;68:380-4.

[32] Glasson MJ, Keay L, Sweeney DF, Wilcox MD. Tolerant and intolerant contact lens wearers show differences in clinical parameters and tear film volume.Invest Ophthalmol Vis Sci 2003;44:5116-24.

[33] Skotnitsky C, Sankaridurg PR, Sweeney DF, Holden BA. General and local contact lens induced papillary conjunctivitis (CLPC). Clin Exp Optom 2002;85:193-7.

[34] Tomlinson A, Blades KJ, Pearce El. What does the phenol red thread test actually measure? Optom Vis Sci 2001;78:142-6.

[35] Jones L, MacDougall N, Sorbara LG. Asymptomatic corneal staining associated with the use of balafilcon silicone-hydrogel contact lenses disinfected with a polyaminopropyl biguanide-preserved care regimen. Optom Vis Sci 2002;79:753-61.

[36] Papas EB, Vajdic CM, Austen R, Holden BA. High-oxygen transmissibility soft contact lenses do not induce limbal hyperaemia. Curr eye Res 1997;16:942-8.

[37] Morgan P, Brennan N. Evaluating corneal oxygenation during lens wear. Contact Lens Spectrum 2007;22:6-13.

[38] Morgan PB, Efron N. Comparative clinical performance of two silicone hydrogel contact lenses for continuous wear. Clin Exp Optom 2002;85:183-92.

[39] Goldberg EP, Bhatia S, Enns JB. Hydrogel contact lens-corneal interactions: a new mechanism for deposit formation and corneal injury. CLAO J. 1997;23:243-8.

[40] Nichols KK, Nichols JJ, Lynn Mitchell G. The relation between tear film tests in patients with dry eye disease. Ophthalmic Physiol Opt. 2003;23:553-60.

[41] Nichols KK, Nichols JJ, Mph M, Mitchell GL. The lack of association between signs and symptoms in patients with dry eye disease. Cornea. 2004;23:762-70.

[42] Guillon M, Maissa C. Bulbar conjunctival staining in contact lens wearers and non contact lens wearers and its association with symptomatology. Contact lens and Anterior Eye 2005;28:67-73.

[43] Begley C, Chalmers R, Abetz L, Venkataran K, Mertzanis P, Caffery B, Snyder C, Edrington $\mathrm{T}$, Nelson $\mathrm{D}$, Simpson $\mathrm{T}$. The relationship between habitual patient related symptoms and clinical signs among patients with dry eye of varying severity. Invest Ophthalmol Vis Sci 2003;44:4753-61.

[44] Thompson B, Collins MJ, Hearn G. Clinical interpersonal communication skills and contact lens wearers' motivation, satisfaction and compliance. Optom Vis Sci 1990;67:6738. 
[45] Jones LA, Walline JJ, Gaume A, Rah MJ, Manny RE, Bernsten DA, Chitkara M, Kim A, Quinn N. Purchase of contact lenses and contact-lenses-related symptoms following the Contact Lenses in Pediatrics (CLIP) Study. Contact Lens Ant Eye 2009;32:157-63. [46] Ramamoorthy P, Sinnott LT, Nichols JJ. Contact lens material charactristics associated with hydrogel lens dehydration. Ophthalmic Physiol Opt 2010;30:160-6.

[47] Ramamoorthy P, Sinnott LT, Nichols JJ. Treatment, material, care and patient-related factors in contact lens-related dry eye. Optom Vis Sci 2008;85:764-72.

[48] Riley C, Young G, Chalmers R. Prevalence of ocular surface symptoms, signs and uncomfortable hours of wear in contact lenses: the effect of refitting with daily wear silicone hydrogel lenses (senofilcon A). Eye Contact Lens 2006;32:281-6.

[49] Pritchard N, Fonn D,Beazeau D Discontinuation of contact lens wear: a survey. International Contact Lens Clinic 1999;26:157-62. 